\title{
Early growth and home environment are associated with cognitive development in the first year of life of Malaysian infants
}

\begin{abstract}
Background: Undernutrition and poor home environment in the first 2 years of life may have long-term effects on cognitive development. Aims: To determine the influence of linear growth and home environment towards cognitive development in the first year of life of Malaysian infants. Study design: Cross-sectional findings at 12-13 months from a prospective cohort study of infants in Malaysia. Subjects: One hundred fifty-one infants recruited at 2-5 months were visited at home at $12-13$ months $(\mathrm{M}=12.95, \mathrm{SD}=0.98)$. Outcome measures: Maternal and infant measures during pregnancy and at birth were obtained from patient health clinic cards. Weight and length of infants were measured at 12-13 months. Home environment and cognitive development were assessed using HOME inventory and Bayley-III, respectively. Results: About $22.5 \%$ of infants experienced stunting and $14.6 \%$ had low home environment quality. The mean cognitive score was $94.54(\mathrm{SD}=12.00)$. Stunted infants had 7.59 lower cognitive scores than non-stunted infants, while infants with low home environment quality had 12.09 lower cognitive scores than those with high home environment quality. Cognitive scores increased with better home environment quality in both stunted and non-stunted infants, but stunted infants scored lower than non-stunted infants in all conditions. Conclusion: Stunting, a form of chronic undernutrition, and poor home environment may significantly influence cognitive development of infants. Interventions to prevent undernutrition should start as early as during pregnancy and caregivers should be informed on their roles in providing cognitive stimulation within the home environment for their children.
\end{abstract}

Keyword: Undernutrition; Stunting; Home environment; Cognitive development; Malaysian infants 